\title{
Comunicación entre el paciente anciano y el médico
}

Serra Rexach JA. Comunicación entre el paciente anciano y el médico. An Med Interna (Madrid) 2003; 20: 57-58.

Una comunicación de calidad entre el personal sanitario y el paciente es imprescindible para conseguir unos buenos resultados. Así se ha demostrado en lo que respecta a la evolución clínica, la satisfacción del paciente, el uso apropiado de los recursos sanitarios, la sensación de bienestar y la adherencia al tratamiento entre otros.

Este tema toma especial relevancia con el envejecimiento de la población en nuestro medio y en los países de nuestro entorno. Actualmente el $16 \%$ de los habitantes de nuestro país tienen más de 65 años (1) y las proyecciones de población sitúan esta proporción en el 18,7\% para el año 2020 (2). Esto conllevará el que los servicios sanitarios de nuestro país tengan que dar respuesta a las necesidades de salud de más de 8 millones de habitantes con una edad superior a los 65 años.

Los ancianos son el grupo de edad que más utiliza los recursos sanitarios. De entre las distintas enfermedades destacan las del sistema circulatorio que son la primera causa de ingreso hospitalario para los mayores de 65 años según los datos de la Encuesta de Morbilidad Hospitalaria, en la Comunidad de Madrid (51,7/1.000 habitantes mayores de 65 años requieren ingreso hospitalario cada año) (3). Dentro de estas enfermedades hay que destacar a la insuficiencia cardiaca. Casi 11 de cada 1.000 ancianos $(10,7 / 1.000)$ ingresan cada año por esta causa, lo que supone la primera causa de hospitalización en mayores de 65 años, totalizando un 5\% de todos los ingresos hospitalarios en este grupo de edad (4).

La prevalencia de insuficiencia cardiaca en el anciano varía según el grupo de población que se analice (5). En el estudio Framingham en ancianos mayores de 80 años se describe una prevalencia de $7 \%$ para varones y $8 \%$ para mujeres. Datos similares se describen en un estudio realizado en Rochester (Estados Unidos) en ancianos mayores de 75 años. En otro estudio realizado en varones de 67 años en Goteborg (Suecia), con unos criterios diagnósticos menos estrictos, la prevalencia fue de $13 \%$. Otros trabajos realizados en distintas ciudades europeas sugieren una prevalencia que varía entre el 3 y el 13\%. Los datos referentes a población española son escasos. En un estudio realizado revisando las historias clínicas de los ancianos de un centro de atención primaria la prevalencia global fue de $6,3 \%$ (4,5\% en menores de 75 años y $8,5 \%$ en los mayores de 75 años) (6).

La comunicación con los pacientes ancianos plantea dificultades por varias circunstancias que pueden interferir (7). Es frecuente que en los ancianos coincidan varias enfermedades crónicas lo que hace que realizar la historia clínica lleve más tiempo de lo normal. También los tratamientos suelen ser más numerosos y en ocasiones difíciles de comprender. Además el deterioro cognitivo con una prevalencia alrededor del $5 \%$ entre los mayores de 65 años y alrededor del $30 \%$ en los mayores de 90 años, afecta de una manera notable la comprensión de las instrucciones sanitarias. Los déficit sensoriales especialmente auditivo y visual producen un tremendo impacto en la interacción entre el profesional sanitario y el paciente. Muchos pacientes ancianos tienen limitaciones funcionales que les impiden realizar independientemente muchas actividades de la vida diaria que pueden afectar el adecuado cumplimiento del tratamiento: abrir el frasco de comprimidos, administrar la dosis adecuada, tragar los comprimidos etc.

En el presente número de la revista se publica un interesante trabajo sobre la información que tienen los ancianos con insuficiencia cardiaca (8). Los autores realizan una entrevista a 75 pacientes mayores de 65 años que ingresan consecutivamente en un servicio de medicina interna. El cuestionario trataba de determinar el grado de conocimiento sobre distintas variables relacionadas con la insuficiencia cardiaca.

Referente a las medidas higiénico-dietéticas más del $80 \%$ de los ancianos entrevistados conocían los beneficios de la restricción de sal, el ejercicio moderado y el control del peso. Sin embargo solo el $37 \%$ conocían los beneficios del control de la diuresis y un $24 \%$ los de la ingesta hídrica moderada. El conocimiento de los síntomas era mucho mejor: Casi el 100\% sabían que debían consultar con el médico por disnea o edemas en miembros inferiores. Sin embargo el conocimiento sobre el tratamiento y las reacciones adversas era peor. Sólo el $48 \%$ sabía que no podía consumir anti-inflamatorios no esteroideos sin consultar con su médico y un 54\% no sabía que los inhibidores del enzima conversor de la angiotensina (IECAs) estaban indicados por la insuficiencia cardiaca. Sólo el $22 \%$ relacionaba los calambres con el tratamiento diurético. El $65 \%$ no relacionaba las náuseas y la inapetencia con la digoxina y el $80 \%$ no relacionaba los mareos y la tos con el tratamiento con IECAs. Es de destacar que para este estudio fueron excluidos aquellos ancianos con algún tipo de alteración cognitiva que pudiera interferir con la comprensión de las preguntas, por lo que en población anciana general los datos todavía deberían ser más llamativos.

Un estudio recientemente publicado, describe resultados similares (9) con un escaso conocimiento de los pacientes de la medicación para la insuficiencia cardiaca, la monitorización del peso, y los síntomas.

Los trabajos que analizan la relación entre el paciente anciano y el médico son escasos, aunque cada vez se publican más. 
Algunos de ellos se centran en la influencia de una relación adecuada entre profesional y paciente con unos buenos resultados terapéuticos. Coe et al (10) grabaron las visitas entre el médico, paciente y cuidador para investigar las estrategias que el médico puede utilizar para mejorar la adherencia del paciente al tratamiento. Encontraron que frecuentemente el paciente y el cuidador no entendían correctamente la medicación incluso aunque el médico repitiera la información y las instrucciones. El médico a menudo dirigía la conversación al cuidador, en lugar de al paciente y resultó de utilidad conocer el régimen de vida del enfermo para adaptar el horario de la medicación. Rost y Roter estudiaron como la interacción entre el médico y el paciente anciano influye en la comprensión del tratamiento y de las recomendaciones en el cambio de estilo de vida y recomiendan revisar al final de la visita estos dos aspectos (11).

Lo importante ahora es conocer que podemos hacer para mejorar la relación entre el personal sanitario y los pacientes para conseguir una mayor adherencia a nuestras recomendaciones y así una mejor evolución de las enfermedades (7). Se puede incidir en varios aspectos: tener en cuenta el punto de vista del paciente, considerar los aspectos psicosociales, valorar las limitaciones sensoriales y funcionales, implicar a los cuidadores en el diagnóstico y en el plan de cuidados y garantizar la continuidad de los cuidados integrando al equipo de atención primaria.

Para entender el punto de vista del enfermo es preciso valorarlo individualmente. No hay dos pacientes iguales. Cada persona tiene sus propias creencias, valores y prioridades que deben tenerse en cuenta a la hora de hacer recomendaciones. Si no las compartimos con ellos es muy posible que no se cumplan correctamente sobre todo si entran en conflicto entre ellas. Es preciso dedicar un tiempo durante la entrevista a estos aspectos, especialmente a la hora de explicar las recomendaciones terapéuticas. Dejar hablar al paciente, siempre es útil.

La valoración de los pacientes ancianos no debe olvidar los aspectos psicosociales que influyen de la misma manera que las enfermedades "tradicionales" en su estado de salud. El médico debe ser capaz de crear el ambiente adecuado, durante la consulta, para que el paciente se sienta confortable para hablar de problemas que puede padecer como soledad, depresión, abuso, temor a la muerte, pérdida de memoria, incontinencia, consumo de alcohol, disfunción sexual etc. Para esto, el paciente debe confiar plenamente en el médico y tener la seguridad de que todo lo que se habla es confidencial. Además debe percibir que hay tiempo suficiente para poder comunicarse. El médico debe evitar hacer juicios de valor, aunque las opiniones del paciente no coincidan con las suyas.

También en los ancianos hay que valorar sistemáticamente la capacidad sensorial y funcional. Si el paciente no oye bien,

\section{Bibliografía}

1. Censos de Población y Viviendas 2001. Instituto Nacional de Estadística. Diciembre 2002. Disponible en www.ine.es.

2. Proyecciones de la Población de España calculadas a partir del Censo de Población de 1991. Evaluación y Revisión. Total nacional 1990-2050. Comunidades autónomas y provincias 1990-2005. Instituto Nacional de Estadística. Octubre 2001.

3. Encuesta de Morbilidad Hospitalaria de 1999 del Instituto Nacional de Estadística y el Padrón Continuo de 1999 de la Comunidad de Madrid.

4. Rodríguez-Artalejo F, Guallar-Castillón P, Benegas Benegas JR, del Rey Calero J. Trends in hospitalization and mortality for heart failure in Spain, 1980-1993. Eur Heart J 1997; 18: 1771-1779.

5. Schocken DD. Epidemiologic and risk factors for heart failure in the elderly. Clin Geriatr Med 2000; 16: 407-417.

6. Gallego Catalán JA, García Domínguez S, Anaya Casbas MT, Álvarez difícilmente podrá seguir de una manera adecuada las instrucciones que le demos. Cuando tenemos en cuenta estas limitaciones y las corregimos o las mejoramos, la comprensión de las instrucciones aumenta. Lo mismo ocurre con la capacidad funcional. Hay que acostumbrarse a incluir en la historia clínica la valoración del estado físico (capacidad de realizar las actividades básicas e instrumentales de la vida diaria) y el estado mental (capacidad cognitiva y afectiva) para conocer más en profundidad al paciente y poder ajustar las recomendaciones a su situación real y mejorar así su cumplimiento.

En la atención de los mayores los cuidadores juegan un papel esencial, especialmente en aquellos con algún grado de dependencia. Es preciso contar con su colaboración para realizar una adecuada historia clínica y su implicación en el cumplimiento del tratamiento es primordial. El trabajador social puede ayudarnos a conocer la situación social de los pacientes, pero debemos trabajar en perfecta coordinación con estos profesionales y no de una manera aislada.

Garantizar la continuidad de los cuidados es imprescindible para un correcto tratamiento de los ancianos. El paciente puede ser visto en las unidades de agudos o en las consultas externas del hospital, pero la mayor parte del tiempo está bajo la supervisión del equipo de atención primaria. Además con frecuencia participan varios especialistas en el manejo del paciente y también este cambia de domicilio y por tanto de equipo de atención primaria. Por todo esto, no es extraño que un paciente anciano sea atendido por muchos médicos distintos al cabo del año, incluso para una misma patología. Si no existe una buena coordinación el incumplimiento del tratamiento está garantizado. Un adecuado informe clínico puede ayudar. Pero es imprescindible instaurar unos adecuados cauces de comunicación entre los distintos médicos y el paciente, que sean rápidos y cómodos. Una comunicación fluida evita incumplimientos terapéuticos, reacciones adversas a medicamentos, visitas a urgencias, ingresos hospitalarios etc. El teléfono parece la opción más adecuada y los modernos sistemas de comunicación (internet) están llamados a desempeñar un papel primordial en un futuro inmediato.

En conclusión, establecer una adecuada relación entre el paciente y el equipo sanitario es la primera condición para una buena evolución clínica. Por esto debemos demandar poder disponer del tiempo adecuado para ello y conocer las técnicas para mejorarla.

\section{J. A. SERRA REXACH}

Departamento Atención Sanitaria. Hospital Gregorio Marañón. Madrid
Pardo JL, Aznar Jiménez R, Córdoba García R. Epidemiología de la insuficiencia cardiaca en el anciano. Atención Primaria 1994; 14: 54-56.

7. Adelman RD, Greene MG, Ory MG. Communication between older patients and their physicians. Clin Geriatr Med 2000; 16:1-24.

8. García Gómez P, Montoto C, Reyes N, García Sánchez I, Agudo P. ¿Qué información tienen los pacientes ancianos con insuficiencia cardiaca sobre su tratamiento. An Med Interna (Madrid) 2003; 20: 59-62.

9. Artinian NT, Magnan M, Christian W, Lange MP. What do patients know about their heart failure? Appl Nurs Res 2002; 15: 200-208.

10. Coe RM, Prendergast CG, Psathas G. Strategies for obtaining compliance with medications regimens. JAGS 1984; 21: 589-594.

11. Rost K, Roter D. Predictors of recall of medications regimens and recommendatios for lifestyle change in elderly patients. Gerontologist 1987; 27: 510-515. 